\title{
Brain abscess formation at the site of intracerebral hemorrhage secondary to central nervous system vasculitis
}

\author{
Case report
}

\author{
Shervin R. Dashti, M.D., Ph.D., Humain Baharvahdat, M.D., Eric Sauvageau, M.D., \\ Steven W. Chang, M.D., Michael F. Stiefel, M.D., Min S. Park, M.D., \\ Robert F. SPETZler, M.D., AND Nicholas C. BAMbaKidis, M.D. \\ Division of Neurological Surgery, Barrow Neurological Institute, St. Joseph's Hospital and Medical \\ Center, Phoenix, Arizona
}

\begin{abstract}
$\checkmark$ Brain abscess is a rare but very dangerous neurosurgical lesion. Prompt diagnosis and emergency surgical evacuation are the hallmarks of therapy. Brain abscess following ischemic and hemorrhagic stroke is a rare entity. These cases are often preceded by episodes of bacteremia, sepsis, and local infection. The authors report the case of a 30-year-old woman who presented with a cerebral abscess at the site of a recent intraparenchymal hemorrhage.
\end{abstract}

(DOI: $10.3171 /$ FOC/2008/24/6/E12)

KEY WORDS • brain abscess • central nervous system • vasculitis

I NTRAPARENCHYMAL hemorrhage complicated by brain abscess is a very rare condition. We present such a case, in which the hemorrhage was thought to be secondary to vasculitis. The difficulty in diagnosis and the theories about the origins of this phenomenon are discussed.

\section{Case Report}

History and Examination. This patient, a 30-year-old woman, presented with sudden-onset lethargy, aphasia, and right-sided weakness. Her medical history was noncontributory, whereas her social history was significant for 1 pack per day of cigarettes and for alcohol abuse. The patient's blood pressure was 130/75 on admission, and her temperature was $37.4^{\circ} \mathrm{C}$. The white blood cell count, platelets, and results of coagulation studies were in the normal range. Results of urine toxicology studies were negative.

Neuroimaging Studies. Admission CT scans of the head revealed a large left basal ganglia intraparenchymal hemorrhage (Fig. 1). Results of an MR image of the brain were consistent with a left basal ganglia hemorrhage without

Abbreviations used in this paper: $\mathrm{BBB}=$ blood-brain barrier; $\mathrm{CT}=$ computed tomography; $\mathrm{MR}=$ magnetic resonance. abnormal enhancement. Four-vessel cerebral angiography was performed to rule out an underlying arteriovenous malformation. The angiogram revealed several foci of narrowing in bilateral anterior cerebral artery and middle cerebral artery distributions, which was consistent with vasculitis (Fig. 2). The angiogram demonstrated no other vascular abnormalities.

Initial Conservative Treatment. The patient was treated conservatively with supportive care. She was in stable condition and was transferred out of the intensive care unit on posthemorrhage Day 8 . She had moderate hemiparesis and mild aphasia at that time. Because of the angiographic evidence of vasculitis, the decision was made to confirm this finding by performing a meningeal and brain biopsy on posthemorrhage Day 10, which was done through a right frontal craniotomy. Specimens of right frontal dura mater as well as cerebral cortex with the overlying meninges were sent for pathological analysis. Pathological findings were consistent with nonspecific changes, with microglial activation, and without definite evidence for vasculitis.

Posttreatment Course. The patient made a slow but progressive improvement in her mental status and right-sided weakness. She was discharged to an acute rehabilitation facility 1 week later. She returned to the hospital 30 days after her intraparenchymal hemorrhage with new onset of seizure followed by a decreased level of consciousness. 


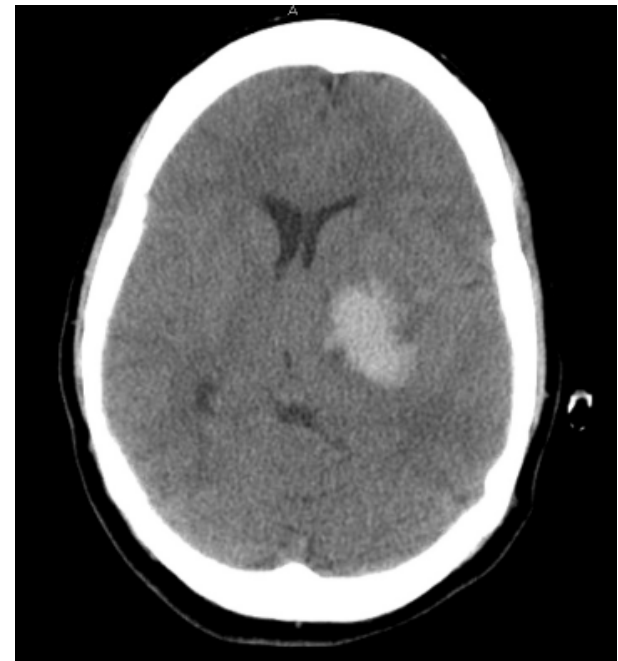

FIG. 1. Axial CT scan of the head obtained without contrast agent, showing a large left basal ganglia hemorrhage.

Before this event, she had been following basic commands with right hemiparesis. A follow-up CT scan of the head revealed a large hypodense lesion in the left basal ganglia with significant surrounding edema (Fig. 3A). The MR imaging of the brain was repeated, and showed interval development of a well-defined, large left hemispheric and brainstem ring-enhancing lesion (Fig. 3B). There was significant surrounding vasogenic edema, with mass effect and midline shift (Fig. 3C). The appearance of the lesion was highly suggestive of cerebral abscess.

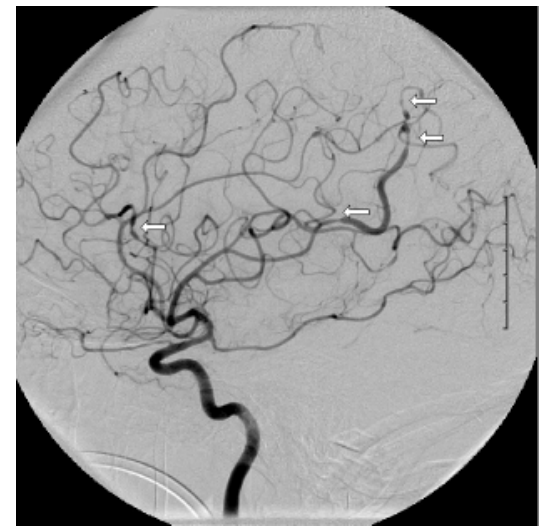

FIG. 2. Cerebral angiogram, left internal carotid artery injection, lateral view, arterial phase. White arrows denote the several focal areas of narrowing in the intracranial arteries.

Emergency Surgery and Postoperative Course. Due to these findings, the patient was taken to the operating room for emergency treatment, and a left frontal craniotomy was performed for evacuation of the lesion. Because of obstructive hydrocephalus, a right frontal ventriculostomy was placed at the same time. A large amount of frank pus was drained. Cultures of this material grew nonlactose fermenting, gram-negative rod bacteria. Final speciation was consistent with Citrobacter koseri. Transesophageal echocardiograms showed no signs of infective endocarditis. The right frontal ventriculostomy could not be discontinued, and it was eventually converted to a right frontal ventriculoperitoneal shunt. The patient was treated with an 8-week

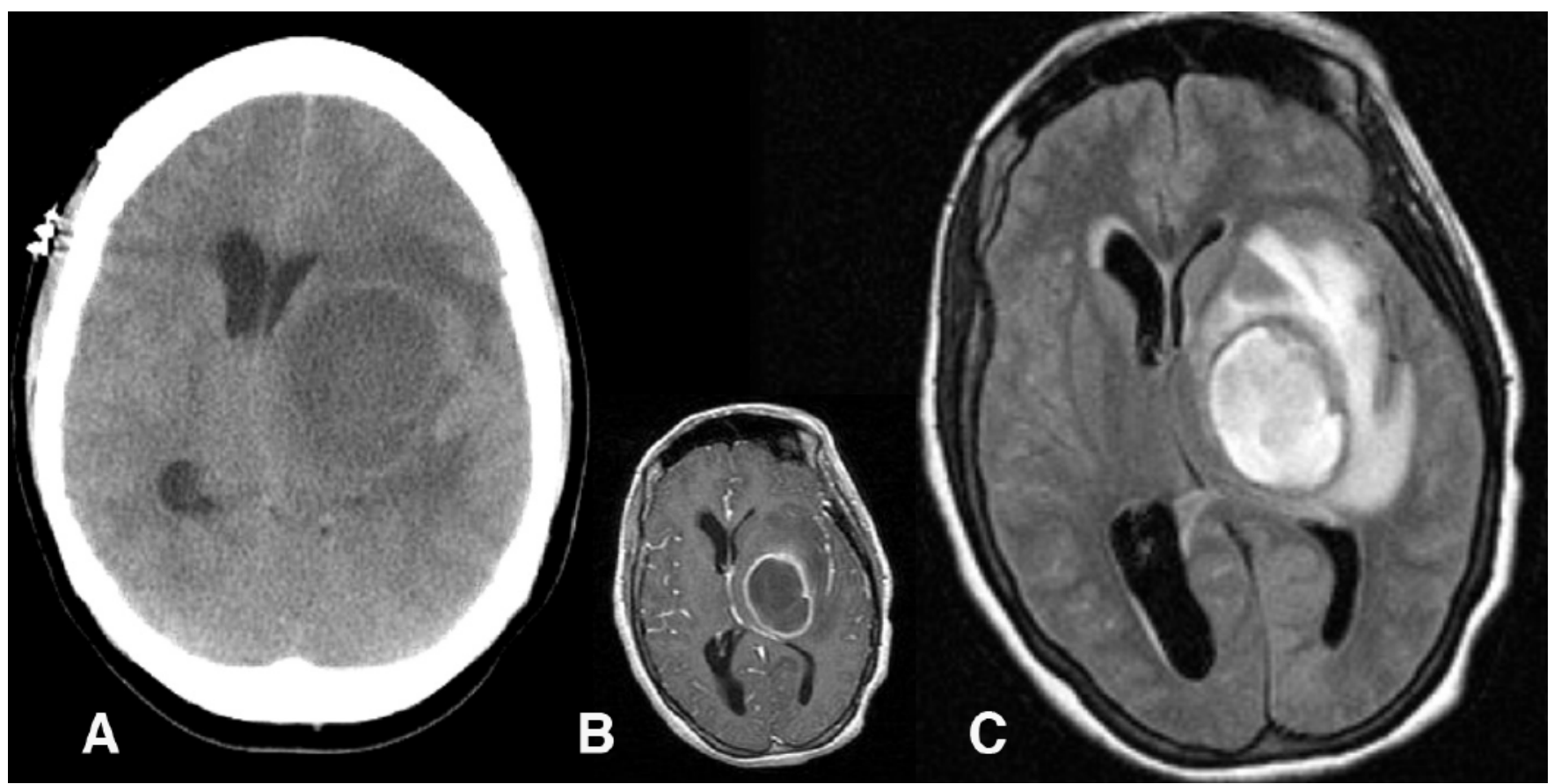

FIG. 3. A. Axial head CT scan obtained without contrast material 30 days after the initial hemorrhage, showing a large hypodense lesion in the left basal ganglia. B. Axial T1-weighted MR image of the brain obtained after addition of gadolinium, revealing a large ring-enhancing cystic lesion suggestive of abscess. C. Axial FLAIR MR image of the brain demonstrating significant surrounding vasogenic edema. 


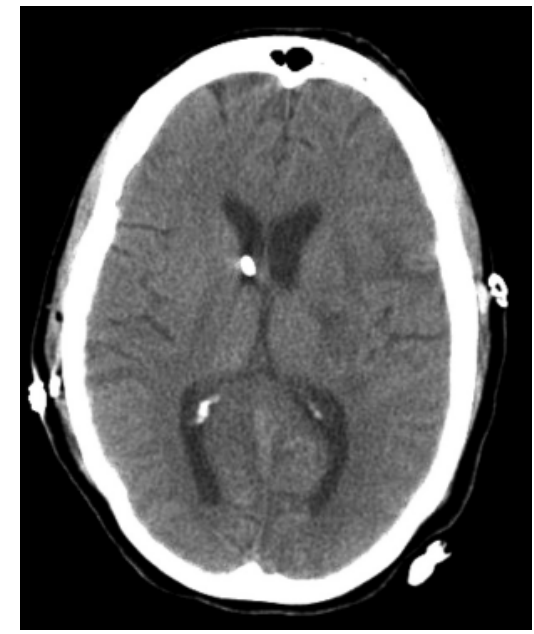

FIG. 4. Axial head CT scan obtained without contrast material after 8 weeks of intravenous antibiotic therapy, showing complete resolution of the abscess.

regimen of intravenous cefepime. The CT scans of the head were repeated, and they showed complete resolution of the abscess (Fig. 4). She was eventually discharged to an inpatient rehabilitation facility. At her last follow-up visit 1 year after the hemorrhage, she was doing well but had moderate right hemiparesis.

\section{Discussion}

Brain abscess is a rare but extremely dangerous neurosurgical lesion. Prompt diagnosis and emergency surgical evacuation are the hallmarks of therapy. Brain abscess is most commonly encountered in the settings of hematogenous spread from a distal focus of infection. Other routes of infection are iatrogenic (that is, postsurgical) or contiguous spread from infected paranasal sinus or dental infection.,6, 9-12,18,20 Absence of a clear source of infection is reported in as many as $40 \%$ of cases. ${ }^{14,15}$

Brain abscess following ischemic and hemorrhagic stroke is a rare entity. These cases are often preceded by episodes of bacteremia, sepsis, and local infection. ${ }^{5,7,8,16} \mathrm{Ab}$ scess formation after intracranial hemorrhage seems to occur mostly via the hematogenous route. This is a likely scenario in our reported case. To our knowledge, a case of intraparenchymal hemorrhage secondary to vasculitis has not been previously reported. It is important to note, however, that the diagnosis of vasculitis was made based on convincing angiographic evidence. Brain biopsy, which is the gold standard test, did not confirm this diagnosis. It is possible that the negative results of brain biopsy were due to sampling error. It is also possible that the appearance of vasculitis on the angiogram was the sequela and not the cause; the dilations in the vasculature may have represented mycotic aneurysms.

Vasculitis consists of granulomatous inflammation and fibrinoid necrosis, which cause disruption of leptomeningeal and parenchymal arteries and veins and potentially lead to hemorrhages. After cerebral hemorrhage, it is thought that the surrounding brain becomes increasingly fragile secondary to breakdown of the BBB. Development of perihematoma edema may involve damage to the vascular endothelium. Iron is a catalyst for lipid peroxidation, and the progressive release of iron from the breakdown of hemoglobin resulting from erythrocyte lysis may contribute to BBB dysfunction. Oxyhemoglobin induced apoptosis through free radical damage to endothelial wall tissue. ${ }^{7}$ The disruption of the BBB may predispose the brain to abscess formation.

An alternative scenario is that our patient's original intraparenchymal hemorrhage was secondary to a preexisting abscess. There have been a handful of reports of hemorrhage from a brain abscess in the literature. . $^{1,4,13,13,17,19,21}$ It has been postulated that intraparenchymal hemorrhage may occur secondary to breakdown of the neovascularization of the abscess cyst wall. In this case, it seems plausible that the blood clot could have hidden evidence of the underlying abscess on the imaging studies, including the contrastenhanced MR image of the brain. Thus, undetected and untreated, the abscess became very large and symptomatic 1 month later, as seen in Fig. 3.

In any case, the possibility of a brain abscess should be taken into consideration following intracerebral hemorrhage when clinical and radiological behavior diverge from the expected course. Brain abscess has a mortality rate of up to $10 \%$, and prompt management with drainage and systemic antibiotics is essential to achieve a favorable outcome. $^{14}$

\section{References}

1. Abbott M, Stern WE: Intracerebral hemorrhage associated with brain abscess. A complication of inappropriate anticoagulation. JAMA 207:1111-1114, 1969

2. Acosta-Feria M, García-Perla A, Infante-Cossío P, BelmonteCaro R, Gutiérrez-Pérez JL: [Intracranial abscess secondary to chronic frontal sinusitis.] Rev Neurol 39:691-693, 2004 (Span)

3. Bach D, Goldenberg MH: Hemorrhage into a brain abscess. J Comput Assist Tomogr 7:1067-1069, 1983

4. Baumann CK: Multiple bilateral cerebral abscesses with hemorrhage. J Neurosci Nurs 29:4-8, 13-14, 1997

5. Beloosesky Y, Streifler JY, Eynan N, Grinblat J: Brain abscess complicating cerebral infarct. Age Ageing 31:477-480, 2002

6. Brook I: Microbiology of intracranial abscesses associated with sinusitis of odontogenic origin. Ann Otol Rhinol Laryngol 115: 917-920, 2006

7. Chen ST, Tang LM, Ro LS: Brain abscess as a complication of stroke. Stroke 26:696-698, 2005

8. Emmez H, Börcek AO, Dogulu F, Ceviker N: Ischemic stroke complicated by a brain abscess: a case report and review of the literature. Turk Neurosurg 17:48-54, 2007

9. Ewald C, Kuhn S, Kalff R: Pyogenic infections of the central nervous system secondary to dental affections-a report of six cases. Neurosurg Rev 29:163-167, 2006

10. Germiller JA, Monin DL, Sparano AM, Tom LW: Intracranial complications of sinusitis in children and adolescents and their outcomes. Arch Otolaryngol Head Neck Surg 132:969-976, 2006

11. Glickstein JS, Chandra RK, Thompson JW: Intracranial complications of pediatric sinusitis. Otolaryngol Head Neck Surg 134: 733-736, 2006

12. Hakim HE, Malik AC, Aronyk K, Ledi E, Bhargava R: The prevalence of intracranial complications in pediatric frontal sinusitis. Int J Pediatr Otorhinolaryngol 70:1383-1387, 2006

13. Lee KS, Bae WK, Bae HG, Doh JW, Yun IG: Brain abscess from a ganglionic hemorrhage-a case report. J Korean Med Sci 9: 259-263, 1994 
14. Mamelak AN, Mampalam TJ, Obana WG, Rosenblum ML: Improved management of multiple brain abscesses: a combined surgical and medical approach. Neurosurgery 36:76-86, 1995

15. Moss SD, McLone DG, Arditi M, Yogev R: Pediatric cerebral abscess. Pediatr Neurosci 14:291-296, 1988

16. Nakai K, Yamamoto T, Yasuda S, Matsumura A: Brain abscess following intracerebral haemorrhage. J Clin Neurosci 13:10471051, 2006

17. Orita T, Fujii M, Hayashi M, Fudaba H, Aoki H: Brain abscess with hemorrhage. Neuroradiology 29:576-577, 1987

18. Ulivieri S, Oliveri G, Filosomi G: Brain abscess following dental procedures. Case report. Minerva Stomatol 56:303-305, 2007

19. Umezawa R, Isozumi K, Komatsumoto S, Fukuuchi Y, Inaba M: [A case of cerebral putaminal hemorrhage complicating a brain abscess proved by craniotomy.] Rinsho Shinkeigaku 44: 372-374, 2004 (Jpn)

20. Wolf J, Curtis N: Brain abscess secondary to dental braces. Pediatr Infect Dis J 27:84-85, 2008

21. Zingale A, Chiaramonte I, Albanese V, Tropea R: Infected cerebral hemorrhage by ruptured infectious anterior communicating artery giant aneurysm in a patient with $\mathrm{T}$ cell acute lymphoblastic leukemia. Case report. J Neurosurg Sci 41:395-400, 1997

Manuscript submitted February 16, 2008.

Accepted March 3, 2008.

Address correspondence to: Nicholas Bambakidis, M.D., 2910

North Third Avenue, Phoenix, Arizona 85013. email: Nicholas Bambakidis@bnaneuro.net. 